\title{
Fast Non-Parametric Estimation of Outbreak Growth from Cumulative Incidence Applied to the Current Ebola Epidemic
}

\author{
Thomas House, School of Mathematics, University of Manchester, M13 9PL, UK.
}

\begin{abstract}
A method is presented that works with cumulative incidence data to provide a real-time estimate of the growth rate of an outbreak, without assuming any particular disease dynamics, and this is applied to the current Ebola outbreak.
\end{abstract}

\section{Working with Cumulative Incidence Data}

During an ongoing outbreak, data are often not available at the level of detail that would be ideal, and in fact often the only publicly available data is on cumulative incidence - i.e. the times that new cases became symptomatic, but not recovery or infection times.

Previous work on Ebola has in fact required much more data than cumulative incidence to perform useful modelling $[7,5,2,1]$, however the question is then posed as to what can be done with cumulative incidence data while bearing in mind the limitations of naive approaches [4].

In this paper I outline an approach to cumulative incidence data that is part of a general framework I am developing called Time-Asymmetric Conjugate Statistical (TACS) learning. One paper has been submitted [3] on a specific, Bernoulli, case of this procedure. A more comprehensive manuscript applying the approach to many different datasets and providing careful comparisons with other methods is in preparation, however since this method may be useful to an unfolding public-health crisis I am releasing full mathematical details and code for the approach, together with results on the current Ebola outbreak, with the caveat that the work is somewhat preliminary.

The TACS estimation approach outlined here rests on three main assumptions:

1. The Force of Infection follows a Gamma distribution

2. Bayes' theorem is a good update rule given new information

3. These two ingredients are all that is required

Of these, 1 and 2 are the strongest - the Gamma distribution is quite flexible, and Bayes' theorem is sound - but 3 is weak since ideally we would be able to build in more scientific knowledge. As such, the current approach is in no way a substitute for a full transmission-dynamic model, but is rather a way to 'make the best of' limited data availability.

Despite this, applying the method to data on the current outbreak gives the results below, with the most important being Figure 4, which shows close to zero current and past growth, but very fast growth around two weeks in to the outbreak. Roughly speaking, a significant trend above the red line implies growth, and a significant trend below implies control. 


\section{Mathematical results}

The Gamma distribution has pdf

$$
f(\lambda \mid \alpha, \beta)=\frac{\beta^{\alpha}}{\Gamma(\alpha)} \lambda^{\alpha-1} \mathrm{e}^{-\beta \lambda} .
$$

The probability of observing $y$ new cases over time $\delta t$ if the force of infection is $\lambda$ is Poisson:

$$
\ell(y \mid \lambda, \delta t)=\frac{(\lambda \delta t)^{y} \mathrm{e}^{-\lambda \delta t}}{\Gamma(y+1)} .
$$

Bayes' theorem holds for any conditional densities and states

$$
\pi^{\prime}(\theta \mid \mathbf{y})=\frac{\ell(y \mid \theta) \pi(\theta \mid y)}{\int \ell(y \mid \vartheta) \pi(\vartheta \mid y) \mathrm{d} \vartheta} .
$$

Applying this to our Gamma prior and Poisson likelihood gives that

$$
f\left(\lambda \mid \alpha^{\prime}, \beta^{\prime}\right)=\kappa \lambda^{\alpha^{\prime}-1} \mathrm{e}^{-\beta^{\prime} \lambda}=\kappa \lambda^{y} \mathrm{e}^{-\lambda \delta t} \lambda^{\alpha-1} \mathrm{e}^{-\beta \lambda}=\kappa \lambda^{(\alpha+y)-1} \mathrm{e}^{-(\beta+\delta t) \lambda},
$$

where $\kappa$ is a normalising constant independent of $\lambda$, which gives us the update rules

$$
\alpha^{\prime}=\alpha+y, \quad \beta^{\prime}=\beta+\delta t .
$$

If $\lambda$ is Gamma distributed with time-dependent parameters $\alpha(t)$ and $\beta(t)$, then $X=\ln (\lambda)$ has pdf

$$
g(x \mid \alpha(t), \beta(t))=\frac{\beta(t)^{\alpha(t)}}{\Gamma(\alpha(t))} \mathrm{e}^{\alpha(t) x-\beta(t) \mathrm{e}^{x}}
$$

Integrating gives

$$
\operatorname{mean}(X)=\psi_{0}(\alpha(t))-\ln (\beta(t)), \quad \operatorname{var}(X)=\psi_{1}(\alpha(t)),
$$

where $\psi_{m}$ is the polygamma function of order $m$. If we consider the effective growth rate $r$ to be the derivative of $X$, then we obtain its mean and variance as

$$
\mu_{r}=\dot{\alpha}(t) \psi_{1}(\alpha(t))-\frac{\dot{\beta}(t)}{\beta(t)}, \quad \sigma_{r}^{2}=\dot{\alpha}(t) \psi_{2}(\alpha(t))
$$

where a dot stands for differentiation with respect to time. These can be estimated at observation points as follows.

We assume that our data is composed of sequential observations of cumulative incidence $C_{1}, C_{2}, \ldots, C_{n}$ at time points $t_{1}, t_{2}, \ldots t_{n}$ such that $t_{i}<t_{i+1}, \forall i$. We then write our update rules as functions of time:

$$
\alpha\left(t_{i+1}\right)=\alpha\left(t_{i}\right)+C_{i+1}-C_{i}, \quad \beta\left(t_{i+1}\right)=\beta\left(t_{i}\right)+t_{i+1}-t_{i} .
$$

This leaves open the values $\alpha(0), \beta(0)$. This can be done through maximum likelihood. Firstly we note that

$$
\int \ell(y \mid \lambda t) f(\lambda \mid \alpha, \beta) \mathrm{d} \lambda=\frac{\beta^{\alpha} t^{y} \Gamma(\alpha+y)}{(\beta+t)^{\alpha+y} \Gamma(y+1) \Gamma(\alpha)} .
$$

Then letting $t_{0}=0, C_{0}=0$ we have likelihood

$$
L(\alpha(0), \beta(0))=\prod_{i=0}^{n} \int \ell\left(C_{i+1}-C_{i} \mid \lambda\left(t_{i+1}-t_{i}\right)\right) f\left(\lambda \mid \alpha\left(t_{i+1}\right), \beta\left(t_{i+1}\right)\right) \mathrm{d} \lambda,
$$

and let

$$
\left(\alpha^{*}, \beta^{*}\right):=\operatorname{argmax} L(\alpha(0), \beta(0)) \text {. }
$$

We numerically optimise $L$, then run the update rules at the optimal initial conditions, before estimating derivaties of the moments of the growth rate $r$. 


\section{Funding Statement}

Work supported by the Engineering and Physical Sciences Research Council, Grant Number $\mathrm{EP} / \mathrm{N} 033701 / 1$.

\section{References}

[1] A. Camacho, A. J. Kucharski, Y. Aki-Sawyerr, M. A. White, S. Flasche, M. Baguelin, T. Pollington, J. R. Carney, R. Glover, E. Smout, A. Tiffany, W. J. Edmunds, and S. Funk. Temporal changes in Ebola transmission in Sierra Leone and implications for control requirements: a real-time modelling study, 2015. doi:10.1371/currents.outbreaks.406ae55e83ec0b5193e3085.

[2] B. S. Cooper, M. F. Boni, W. Pan-ngum, N. P. J. Day, P. W. Horby, P. Olliaro, T. Lang, N. J. White, L. J. White, and J. Whitehead. Evaluating clinical trial designs for investigational treatments of Ebola virus disease. PLOS Medicine, 12(4):e1001815, 2015.

[3] T. House, R. Vahid Roudsardi, and L. Dawson. Beta process learning of longitudinally assessed clinical performance in dental education. Submitted, 2018.

[4] A. A. King, M. Domenech de Cellès, F. M. G. Magpantay, and P. Rohani. Avoidable errors in the modelling of outbreaks of emerging pathogens, with special reference to Ebola. Proceedings of the Royal Society of London B: Biological Sciences, 282(1806), 2015.

[5] A. A. Kucharski, A. Camacho, F. Checchi, R. Waldman, R. R. Grais, J.-C. J.-C. Cabrol, S. Briand, M. Baguelin, S. Flasche, S. Funk, W. J. Edmunds, and W. John Edmunds. Evaluation of the benefits and risks of introducing Ebola community care centers, Sierra Leone. Emerging Infectious Diseases, 21(3):393-399, 2015.

[6] C. Rivers. Data for the 2018 Ebola outbreak in the Democratic Republic of Congo. doi:10.5281/zenodo.1246777, 2018.

[7] WHO Ebola Response Team. Ebola virus disease in West Africa - the first 9 months of the epidemic and forward projections. New England Journal of Medicine, 371(16):1481-1495, 2014.

\section{Python Code and Results}

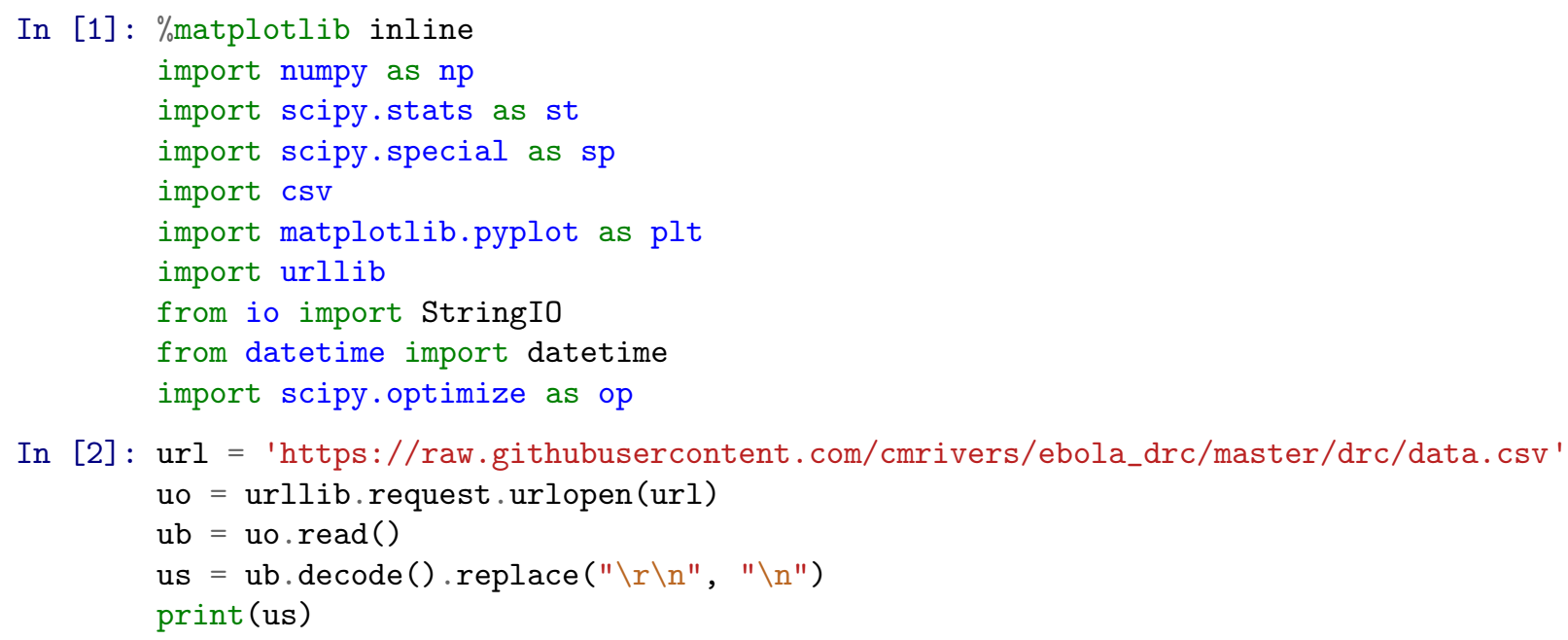


bioRxiv preprint doi: https://doi.org/10.1101/340067; this version posted June 8,2018 . The copyright holder for this preprint (which was not certified by peer review) is the author/funder, who has granted bioRxiv a license to display the preprint in perpetuity. It is made available under aCC-BY 4.0 International license.

event_date,report_date, health_zone, confirmed_cases,new_confirmed,probable_cases,new_probable, suspect_cases, new_suspect, deaths 20180501,20180510, Bikoro, , , , ,21, ,17

20180510, 20180510,Bikoro, , , , , , 9 , , 1

20180511, 20180511, Bikoro, 2, , , , 6, , 0

20180511, 20180511, Iboko, 0, , , , , 6, , 1

20180512,20180512, Bikoro, 2, , , , 6, , 0

20180512, 20180512, Iboko, 0, , , , 6, , 1

20180513, 20180514, Bikoro, 2, 0,18, 0, 12, 0,

20180513, 20180514, Iboko, 0, 0, 3, 0, 5, 2,

20180513, 20180514, Wangata , 0, 0, 0, 0, 2, 0,

20180515, 20180516, Bikoro, 2, 0, 18, 0, 15, 1,

20180515, 20180516, Iboko, 0, 0, 2, 0, 3, 0,

20180515, 20180516, Wangata, 1, 0, 0, 0, 3, 0,

20180515, 20180516, Ntondo, 0, 0, 0, 0, 0, 0,

20180516, 20180517, Bikoro, 13, 0, 19, 1, 4, 0, 21

20180516, 20180517, Iboko, 0, 0, 2, 0, 3, 0, 3

20180516, 20180517, Wangata, 1, 0, 0, 0, 3, 0, 1

20180516, 20180517, Ntondo , 0, 0, 0, 0, 0, 0, 0

20180517, 20180518, Bikoro, 10, 0, 19, 0, 0, 0, 21

20180517,20180518 , Iboko, 3, 0, 2, 0, 4, 4, 3

20180517,20180518 , Wangata , 4, 0, 0, 0,1,1,1

20180518, 20180520, Bikoro, 10, 0, 19, 0, 0, 0, 22

20180518,20180520 , Iboko , 7, 0, 2, 0, 2, 2, 3

20180518, 20180520, Wangata , 4, 0, 0, 0, 2, 1, 1

20180520, 20180521, Bikoro, 10, 0, 19, 0, 0, 0, 22

20180520,20180521 , Iboko, 14, 0, 2, 0, 0, 0, 3

20180520, 20180521, Wangata , 4, 0, 0, 0, 2, 2, 2

20180521, 20180522, Bikoro, 10, 0, 19, 0, 0, 0, 23

20180521, 20180522, Iboko, 14, 0, 2, 0, 6, 6, 3

20180521,20180522 , Wangata , 4 , 0, 0, 0, 3, 2, 1

20180522, 20180523, Bikoro, 10, 0, 12, 1, 2, 2, 16

20180522 , 20180523, Iboko, 16, 2 , 2 , 0, 6 , 4, 3

20180522, 20180523, Wangata , 4, 0, 0, 0, 6, 3, 3

20180523, 20180524, Bikoro, 10, 0, 11, 0, 2, 2, 16

20180523,20180524 , Iboko, 17, 1, 2, 0, 5, 1, 3

20180523, 20180524, Wangata , 4, 0, 0, 0, 1, 0 ,

20180524, 20180525, Bikoro, 10, 0, 11, 0, 3, 3, 16

20180524, 20180525, Iboko, 21, 4, 2, 0, 2, 0, 4

20180524,20180525 , Wangata , 4, 0, 0, 0, 1, 1, 3

20180525, 20180526, Bikoro, 10, 0, 11, 0, 2, 2, 16

20180525, 20180526, Iboko, 21, 0, 2, 0, 2, 0, 6

20180525,20180526 , Wangata , 4, 0, 0, 0, 1, 1, 3

20180525, 20180526, Ntondo, 0, 0, 0, 0,1,1,0

20180526, 20180527, Bikoro, 10, 0,11,0,1,1,16

20180526, 20180527, Iboko, 21, 0, 2, 0, 5, 3, 6

20180526, 20180527, Wangata , 4, 0, 0, 0, 1, 0, 3

20180526, 20180527, Ntondo, 0, 0, 0, 0,1,0,0

20180527, 20180528, Bikoro, 10, 0,11, 0,1,0,16

20180527,20180528 , Iboko, 21, 0, 2, 0, 4, 0, 6

20180527,20180528 , Wangata , 4, 0, 0, 0, 1, 1, 3

20180527, 20180528, Ntondo, 0, 0, 0, 0, 0, 0,0

20180528, 20180529, Bikoro, 10,0,11, 0, 1, 0, 16

20180528, 20180529, Iboko, 21, 0, 2, 0, 1, 1, 6

20180528, 20180529, Wangata , 4, 0, 0, 0, 1, 1, 3

20180529, 20180530, Bikoro, 10, 0,11, 0, 0, 0, 16

20180529,20180530 , Iboko, 22, 1, 2, 0, 3, 3, 6

20180529,20180530 , Wangata , 4 , 0, 0, 0, 1, 0, 3

20180530, 20180531, Bikoro, 10, 0, 11, 0, 0, 0, 17

20180530, 20180531, Iboko, 23, 1, 2, 0, 0, 0, 5

20180530,20180531 , Wangata , 4, 0, 0, 0, 0, 0, 3

20180531,20180601, Bikoro, 10, 0, 11, 0, 3, 3, 17

20180531, 20180601, Iboko, 23, 0, 2, 0, 0, 0, 5

20180531 , 20180601, Wangata , 4, 0, 0, 0, 2, 2, 3

20180601,20180602 , Bikoro, 10, 0, 11, 0, 2, 2, 17

20180601, 20180602, Iboko , 23, 0, 2, 0, 3, 3, 5

20180601,20180602 , Wangata , 4, 0, 0, 0, 2, 2, 3

20180602, 20180603, Bikoro, 10,0,11,0,0,0,17

20180602, 20180603, Iboko, 23, 0, 2, 0, 3, 1, 5

20180602, 20180603, Wangata , 4, 0, 0, 0, 0, 0, 3

20180603, 20180604, Bikoro, 10,0,11,0,5,5,17

20180603,20180604 , Iboko, 23, 0, 2, 0, 0, 0,5

20180603,20180604 , Wangata , 4, 0, 0, 0, 1, 1, 3

In [3]: reader $=$ csv.reader (StringIO(us), delimiter =', ' )

next (reader, None) \#Header

$\mathrm{tb}=\mathrm{np} \cdot \operatorname{array}([])$

$\mathrm{ti}=\mathrm{np} \cdot \operatorname{array}([])$ 
bioRxiv preprint doi: https://doi.org/10.1101/340067; this version posted June 8,2018 . The copyright holder for this preprint (which was not certified by peer review) is the author/funder, who has granted bioRxiv a license to display the preprint in perpetuity. It is made available under aCC-BY 4.0 International license.

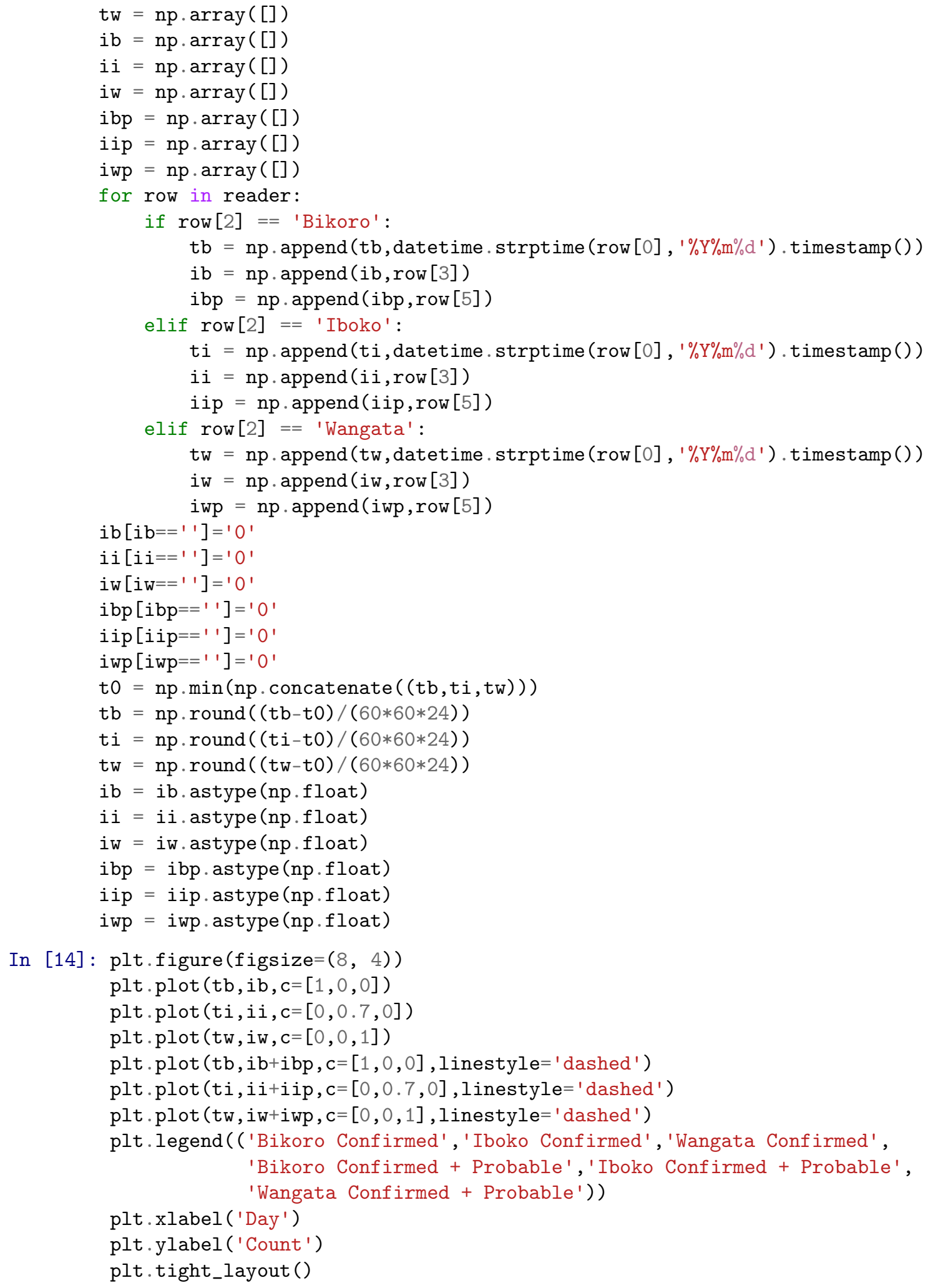

In [14]: plt.figure(figsize=(8, 4))

plt.plot (tb ,ib, $c=[1,0,0])$

plt.plot (ti, ii, $c=[0,0.7,0])$

plt.plot $(t w, i w, c=[0,0,1])$

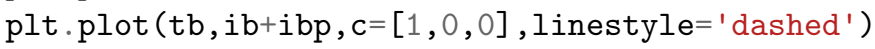

plt.plot (ti, ii+iip, $c=[0,0.7,0], 1$ inestyle=' dashed' $)$

In [5]: \# Add confirmed cases 
bioRxiv preprint doi: https://doi.org/10.1101/340067; this version posted June 8,2018 . The copyright holder for this preprint (which was not certified by peer review) is the author/funder, who has granted bioRxiv a license to display the preprint in perpetuity. It is made available under aCC-BY 4.0 International license.

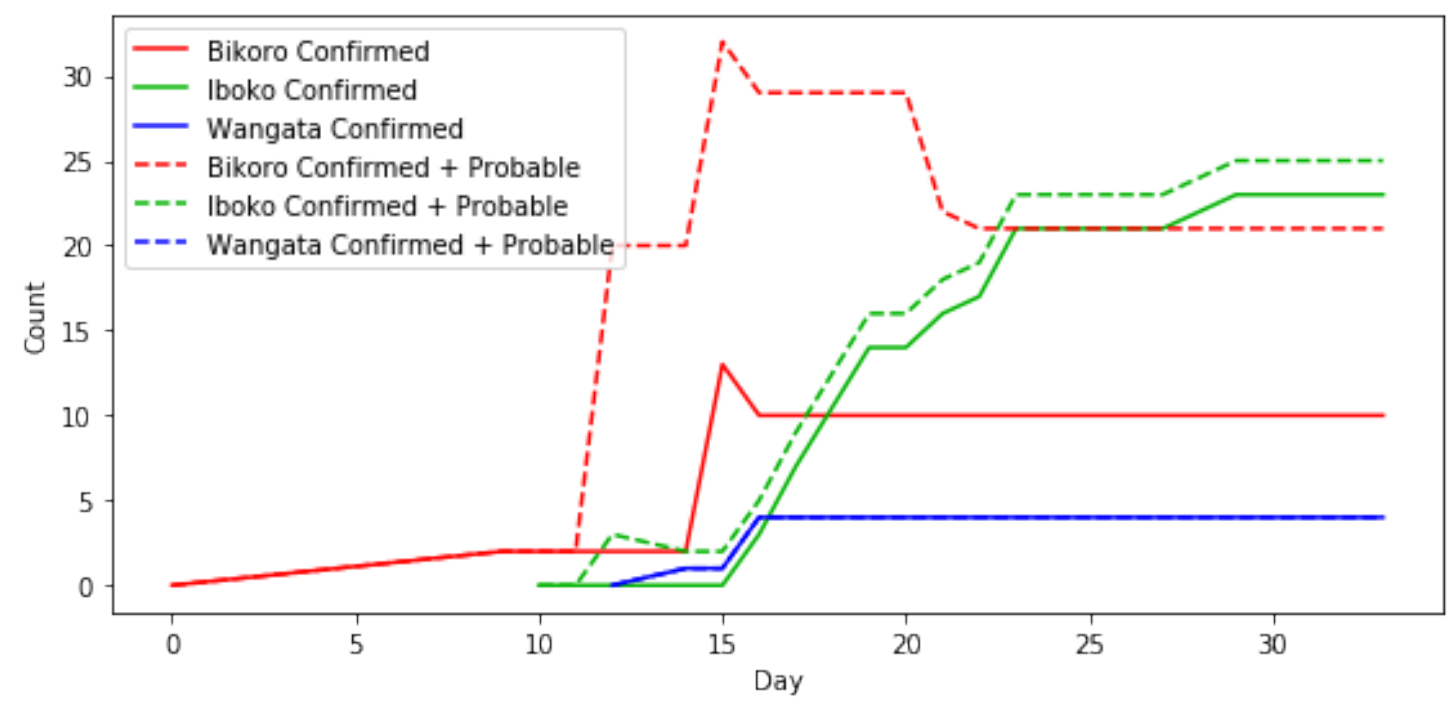

Fig 1: Cases. Confirmed and Probable Cases [6].

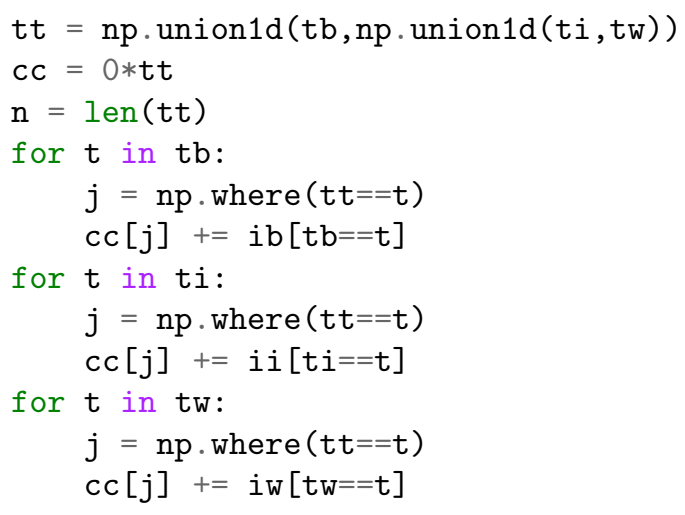

In [6]: \# Remove non-informative points - this assumes no doubly flat lines

$\mathrm{kk}=\mathrm{np} \cdot$ where $(\mathrm{np} \cdot \operatorname{diff}(\mathrm{cc})>0)$

$\mathrm{kk}=\mathrm{np} \cdot \operatorname{append}(\mathrm{kk}, \mathrm{len}(\mathrm{cc})-1)$

$t t=t t[k k]$

$\mathrm{cc}=\mathrm{cc}[\mathrm{kk}]$

$\mathrm{n}=\operatorname{len}(\mathrm{tt})$

In [15]: \# Plot

plt.figure (figsize $=(8,4))$

plt.plot (tt, cc, $c=[0,0,0])$

plt.xlabel ('Day')

plt.ylabel('Cumulative Incidence for Confirmed')

plt.tight_layout()

In [8]: def nllfun(x,tt,cc,nn):

$$
\begin{aligned}
& \mathrm{t}=\mathrm{np} \cdot \mathrm{zeros} \_l i k e(\mathrm{cc}) \\
& \mathrm{y}=\mathrm{np} \cdot \mathrm{zeros} \text { _like }(\mathrm{cc}) \\
& \mathrm{al}=\mathrm{np} \cdot \mathrm{zeros} \text { _like }(\mathrm{cc}) \\
& \mathrm{bt}=\mathrm{np} \cdot \mathrm{zeros} \text { _like }(\mathrm{cc})
\end{aligned}
$$




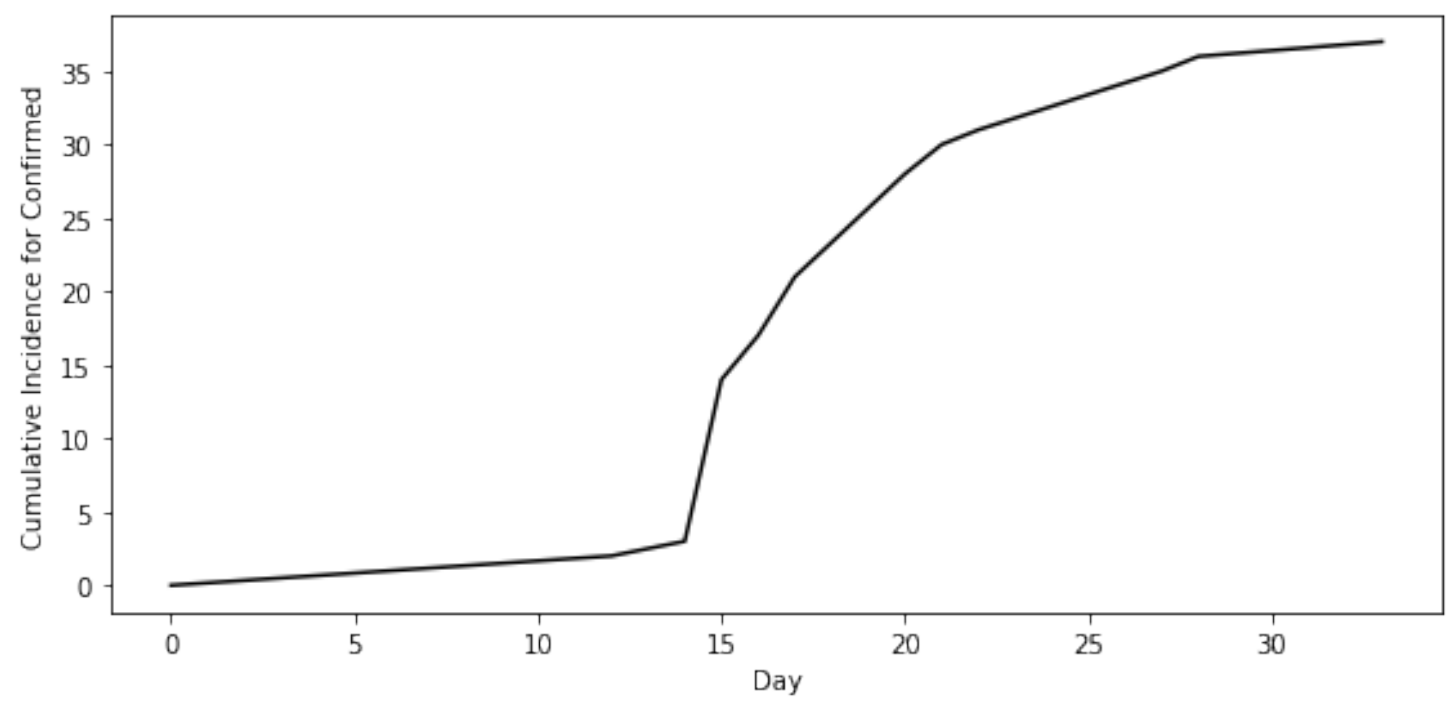

Fig 2: Cumulative Incidence. Assumed cumulative incidence curve.

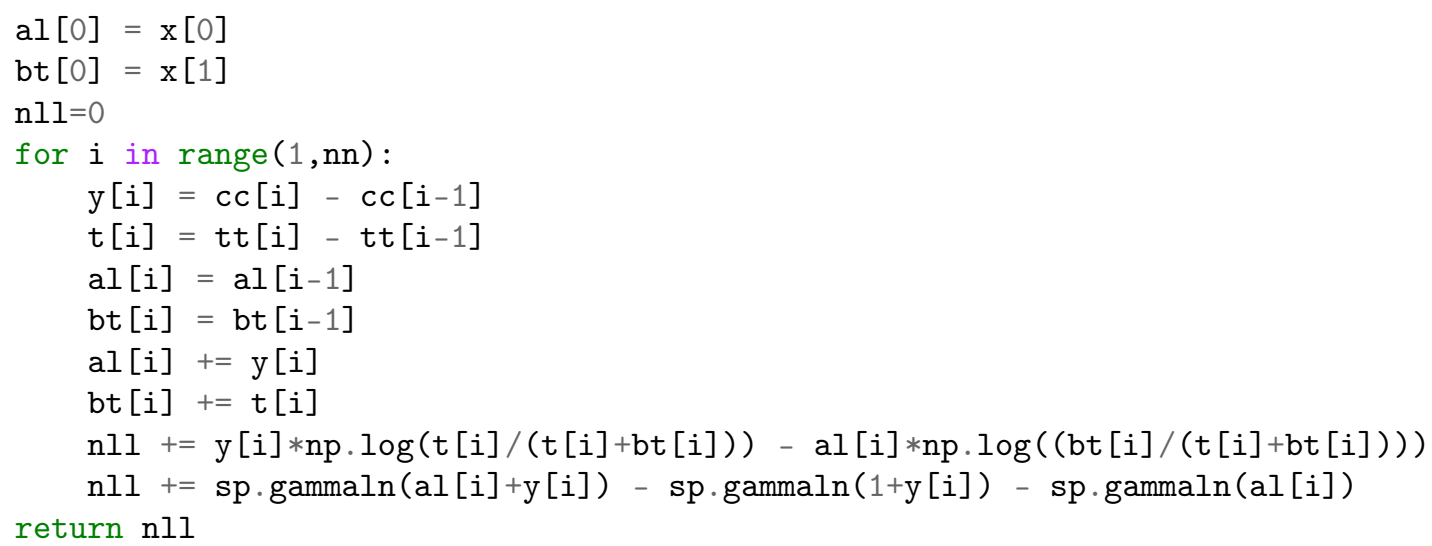

In [9]: \#Optimizer to fit the initial conditions

$\mathrm{nll}=$ lambda $\mathrm{xx}$ : nllfun (np.abs $(\mathrm{xx})$, tt, $\mathrm{cc}, \mathrm{n})$

fout $=$ op.minimize $\left(n l l, n p \cdot \operatorname{array}((1.0,1.0)), \operatorname{method}={ }^{\prime}\right.$ Nelder-Mead' $)$

astar $=n p \cdot a b s($ fout $\cdot x[0])$

bstar $=n p \cdot a b s($ fout $\cdot x[1])$

fout.x

Out [9] : $\operatorname{array}([-3.32222615 e+01,2.33945606 e+08])$

In [10]: $t=n p \cdot z e r o s \_l i k e(c c)$

$\mathrm{y}=\mathrm{np} \cdot \mathrm{zeros} \_$like (cc)

$\mathrm{al}=\mathrm{np} \cdot \mathrm{zeros}_{-}$like $(\mathrm{cc})$

$\mathrm{bt}=\mathrm{np} \cdot \mathrm{zeros} \_$like $(\mathrm{cc})$

lam $=$ np.zeros_like $(c c)$

lal $=$ np.zeros_like $(c c)$

lau $=$ np.zeros_like $(c c)$

$\mathrm{al}[0]=$ astar

bt $[0]=$ bstar

$\operatorname{lam}[0]=$ st.gamma.mean $(\mathrm{al}[0], 0,1 / \mathrm{bt}[0])$

lal $[0]=$ st.gamma $\cdot \operatorname{ppf}(0.025, \mathrm{al}[0], 0,1 / \mathrm{bt}[0])$

lau $[0]=$ st.gamma $\cdot \operatorname{ppf}(0.975, \mathrm{al}[0], 0,1 / \mathrm{bt}[0])$ 


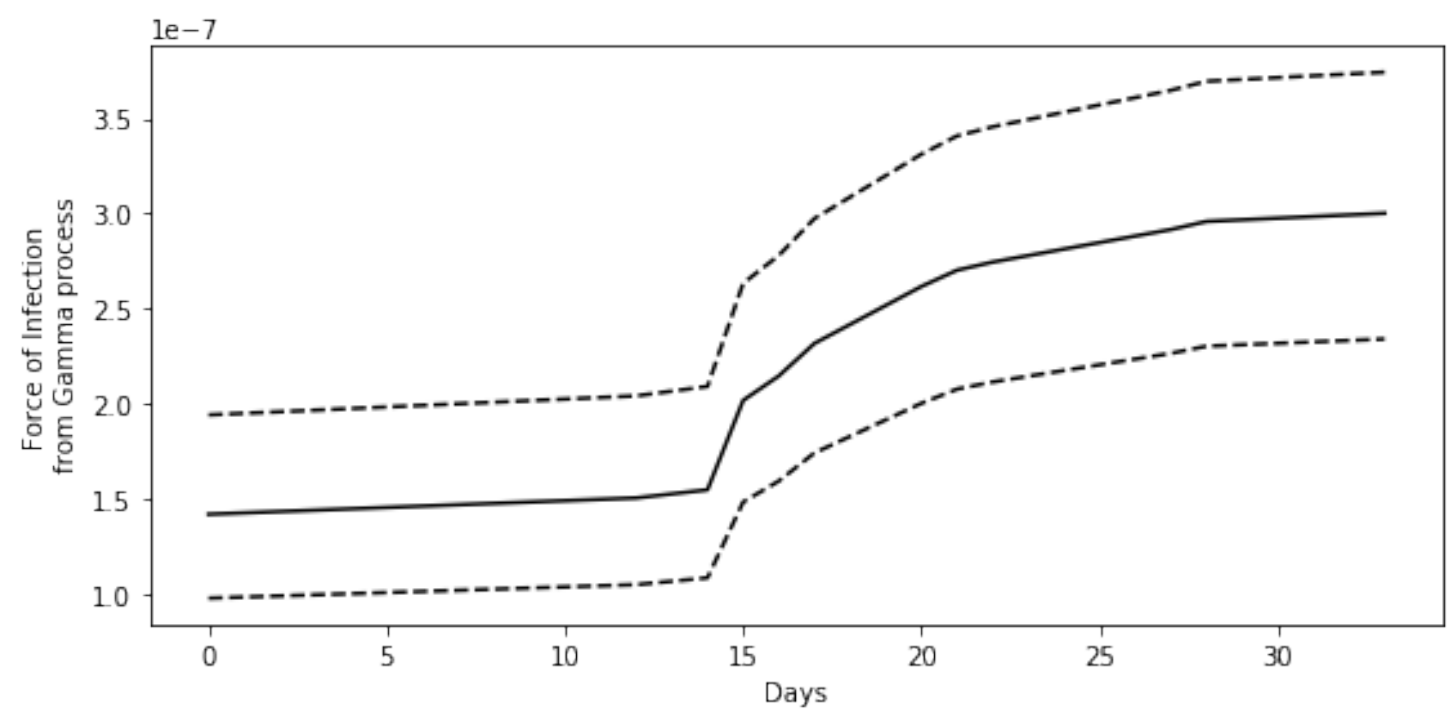

Fig 3: FOI. Inferred Force of Infection, $\lambda$.

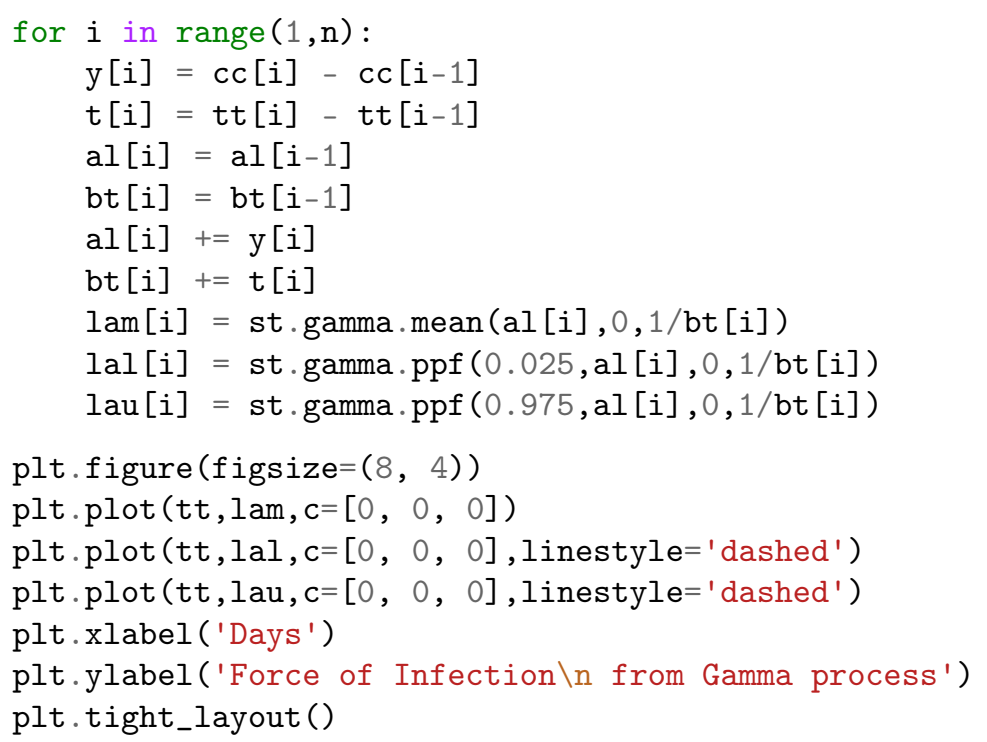

In $[12]: a m=a l$

$\mathrm{bm}=\mathrm{bt}$

$\mathrm{dt}=\mathrm{np} \cdot \operatorname{gradient}(\mathrm{tt})$

$\mathrm{dth}=(\mathrm{np} \cdot \operatorname{gradient}(\mathrm{bm}) /(-1.0 * \mathrm{bm} * * 2)) / \mathrm{dt}$

$\mathrm{da}=($ np.gradient $(\mathrm{am})) / \mathrm{dt}$

$\mathrm{rm}=(\mathrm{dth} * \mathrm{bm})+(\mathrm{sp} \cdot \operatorname{polygamma}(1, \mathrm{am}) * \mathrm{da})$

$\mathrm{rv}=(\mathrm{sp} \cdot \operatorname{polygamma}(2, \mathrm{am}) * \mathrm{da})$

$\mathrm{rs}=\mathrm{np} \cdot \operatorname{sqrt}(-\mathrm{rv})$

In [17]: plt.figure (figsize $=(8,4))$

plt.plot (tt, rm, $c=[0,0,0])$

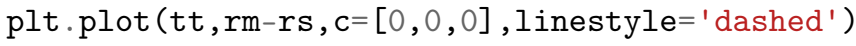

plt.plot (tt, $r m+r s, c=[0,0,0]$, linestyle $=$ ' dashed $\left.^{\prime}\right)$ 
bioRxiv preprint doi: https://doi.org/10.1101/340067; this version posted June 8, 2018. The copyright holder for this preprint (which was not certified by peer review) is the author/funder, who has granted bioRxiv a license to display the preprint in perpetuity. It is made available under aCC-BY 4.0 International license.

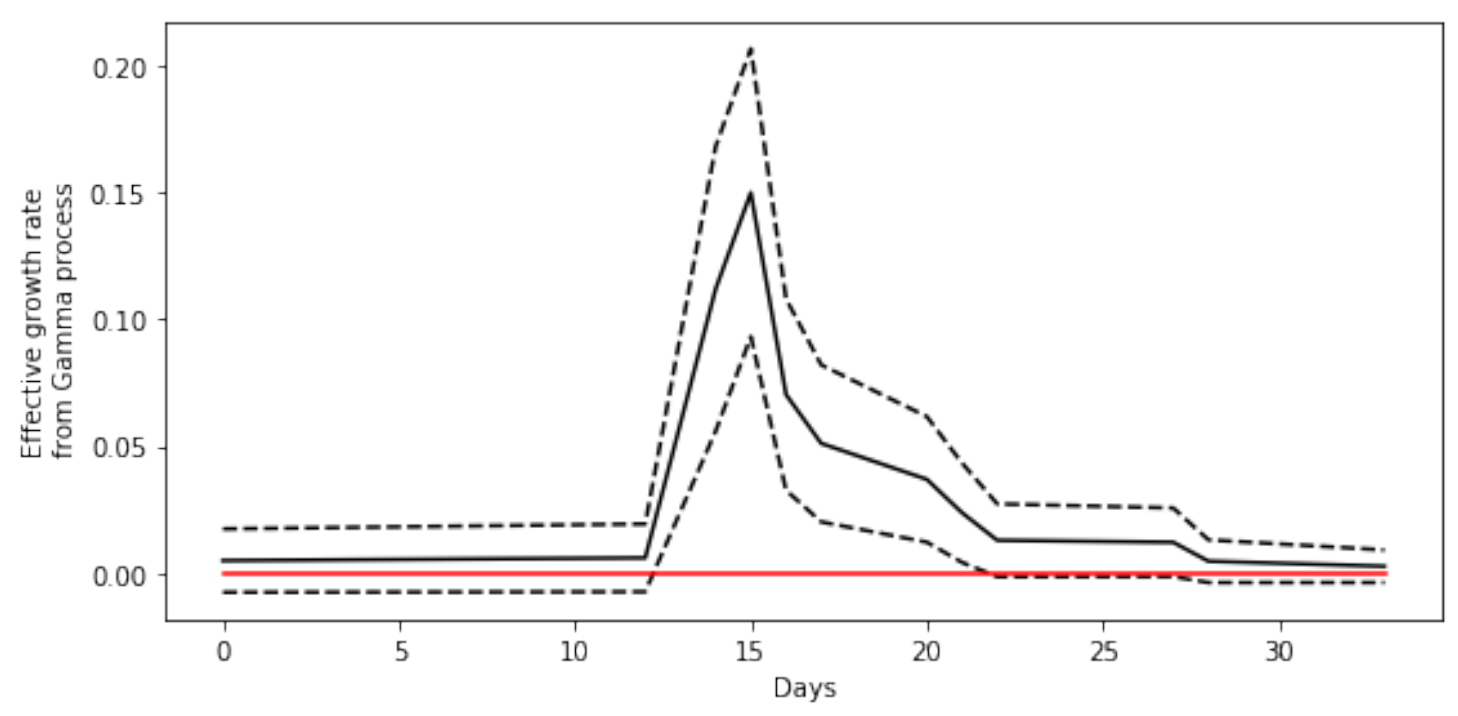

Fig 4: Growth. Inferred growth rate $r$.

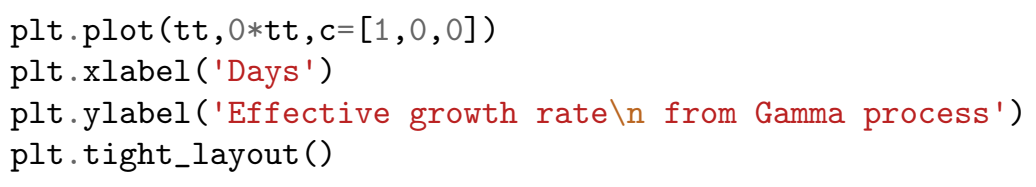

